\title{
Intercellular cannibalism fuels tumor growth
}

\author{
Ernesto Pérez ${ }^{1,2}$ and Andreas Bergmann ${ }^{*, 1}$ \\ Cell Death and Differentiation (2017) 24, 759-760; doi:10.1038/cdd.2017.39; published online 24 March 2017
}

Macroautophagy (hereafter autophagy) is a major catabolic process in eukaryotic cells, which controls quality and quantity of intracellular components, including proteins and organelles. ${ }^{1}$ An essential step during autophagy is the formation of autophagosomes, which deliver cargo such as mis-folded proteins and damaged organelles to lysosomes for degradation (Figure 1). After degradation, amino acids and building blocks of organelles are recycled into the cytoplasm for biosynthetic pathways (Figure 1). Under normal conditions, basal autophagy ensures homeostasis of the cell, but under stress conditions such as starvation, autophagy is strongly induced and promotes the survival of the cell. However, autophagy can also trigger cell death (autophagic cell death), particularly in insects. ${ }^{2,3}$

Genetic screens in yeast have uncovered more than 30 autophagy-related (Atg) genes that are involved in all steps of the autophagic process. ${ }^{4}$ Most Atg genes are conserved between yeast and mammals, and have very similar functions for autophagy in these organisms. For example, Atg8 and its mammalian homolog LC3 encode ubiquitin-like proteins that are conjugated with lipids in the membranes of the autophagosomes, and its punctate appearance in cells can be used as a marker for autophagosome formation. ${ }^{4}$ Atg7 is an essential protein for lipidation of Atg8/LC3 and its genetic inactivation is often used to block autophagy. ${ }^{1}$

Because of its essential biological role, it is not surprising that autophagy is also involved in many pathological conditions, including cancer. ${ }^{1,2}$ Although mutations in core Atg genes are rarely found in human cancer, ${ }^{5}$ autophagy nevertheless influences the outcome of oncogenesis. However, its exact function in cancer is unclear as both tumor-suppressing as well as tumor-promoting activities of autophagy have been reported. ${ }^{1}$ For example, in epithelial tumor models in the fruit fly Drosophila melanogaster, autophagy is required for tumor growth associated with oncogenic mutations of $\operatorname{Ras}\left(\operatorname{Ras}^{V 12}\right)$, but it restricts growth of Notch-driven tumors. ${ }^{6}$ Not only does the oncogene appear to determine the function of autophagy for tumorigenesis, but also the cell type. Activation of autophagy has opposite effects on epithelial vs glia overgrowth induced by expression of YAP/Yorkie, the transcription factor of the Hippo growth control pathway. Epithelial YAP/Yorkie-induced overgrowth can be suppressed by autophagy, while glial overgrowth induced by the same signal is further enhanced by autophagy. ${ }^{6}$
In genetically engineered mouse models (GEMM), best characterized is the role of autophagy in tumors associated with oncogenic mutations of $\mathrm{Kras}^{1}{ }^{1}$ Growth of $\mathrm{Kras}^{\mathrm{G1} D}$-induced non-small-cell lung cancer (NSCLC) and pancreas cancer depend on functional autophagy. ${ }^{1}$ If autophagy is blocked by genetic inactivation of $\operatorname{Atg} 7$ or Atg5, tumors do not develop beyond an initial benign state, which lead to the proposal that autophagy has tumor-suppressing activities for tumor initiation, but is required for efficient and malignant growth during tumor progression of these cancers. ${ }^{1}$ In Kras-driven gliobastoma, autophagy appears to be required for every step in tumor development. ${ }^{1}$ Similarly, autophagy promotes Braf ${ }^{V 600 E}$-driven melanoma. Therefore, it has been proposed that the 'autophagy-addiction' of these tumor types can be exploited for cancer therapy by therapeutic targeting of autophagy. $1^{1}$

In all these examples, autophagy has been experimentally manipulated inside the tumor cells and based on the obtained data, it is generally assumed that autophagy acts autonomously in tumor cells. However, a recent study in Nature, performed in Drosophila, suggested that tumor cells can also trigger non-autonomous autophagy (NAA) in neighboring normal (non-tumor) cells (Figure 1). ${ }^{7}$ In fact, the authors demonstrate that NAA has a much more profound role for tumor growth than autonomous autophagy, although the latter also contributes to final tumor volume.6,7 The authors employed an established epithelial tumor model in Drosophila, in which tumor cells express oncogenic $\operatorname{Ras}^{\mathrm{V} 12}$ and at the same time have also lost apical/basal polarity due to genetic inactivation of the gene scribble (referred to as Ras ${ }^{V 12}$ scrib) (Figure 1). ${ }^{8,9}$ Epithelial $\operatorname{Ras}^{V 12}$ scrib cells fail to respond to stop signals for growth, fail to differentiate and grow into very aggressive, neoplastic tumors. They also invade other tissues, which eventually results in the death of the affected animal. Thus, this model mimics tumor development in human patients and provides valuable information for understanding tumorigenesis.

The authors examined the role of autophagy in the Ras ${ }^{V 12}$ scrib model, and using an Atg8/LC3 autophagy reporter, they surprisingly observed that autophagy is strongly induced in neighboring non-tumor cells, indicating NAA. ${ }^{7}$ To evaluate the significance of autophagy in the Ras ${ }^{V 12}$ scrib model, they performed a number of elegant genetic experiments, in which they specifically eliminated autophagy either in the tumor cells

\footnotetext{
1Department of Molecular, Cell and Cancer Biology, University of Massachusetts Medical School, 364 Plantation Street, LRB419, Worcester 01605, MA, USA *Corresponding author: A Bergmann, Department of Molecular, Cell and Cancer Biology, University of Massachusetts Medical School, 364 Plantation Street, LRB419, Worcester 01605, MA, USA. Tel: +1 508.856.6423; Fax: +1 (508)856.1310; E-mail: andreas.bergmann@ umassmed.edu

${ }^{2}$ Present address: Department of Biology, Austin College, 900N. Grand Ave, Suite 61611, Sherman, TX 75090, USA.
} 


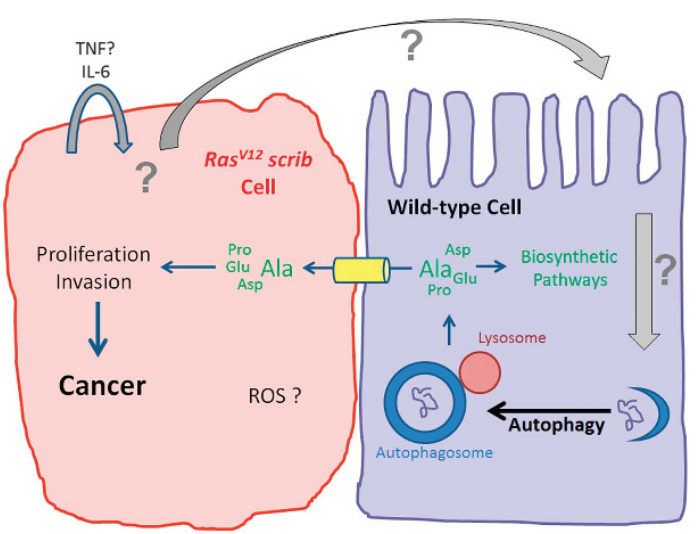

Figure 1 Non-autonomous autophagy (NAA) fuels tumor growth. Ras ${ }^{\text {V12 }}$ scrib tumor cells (left) generate an unknown signal, which triggers NAA in neighboring wild-type cells (right). Released amino acids such as Alanine (Ala), but likely also others, are transported into Ras ${ }^{\mathrm{V} 12}$ scrib tumor cells where they may be used as alternative carbon source for proliferation and invasion, triggering tumor growth

or in the non-tumor environment or both. While they do observe a weak requirement of autophagy tumor-autonomously, a much stronger suppression of tumor growth was observed when autophagy was inactivated in the microenvironment, even when still intact in the tumor itself. ${ }^{7}$ The authors took these experiments a step further and performed allograft transplantation experiments of $\operatorname{Ras}^{V 12}$ scrib tissue into autophagy-competent or autophagy-deficient hosts. The results were clear. Only when the recipient was autophagycompetent, did the transplanted $\operatorname{Ras}^{V 12}$ scrib tissue induce tumor growth, even when the tumor itself was autophagydeficient. ${ }^{7}$ These results strongly suggest that NAA is very critical for efficient growth of Ras ${ }^{V 12}$ scrib tumors.

These observations raised two important questions. First, what are the signals from tumor cells triggering NAA and second what is the function of NAA for growth of Ras ${ }^{V 12}$ scrib tumors? The authors indeed found that TNF and IL-6 cytokines are involved in NAA, but this seems to be an autocrine requirement, at least for IL-6 (Figure 1). ${ }^{7}$ The authors also observed the generation of reactive oxygen species (ROS), but a specific role of ROS for NAA was not established. To answer the second question, the authors considered that autophagy provides nutrients for biosynthetic pathways of the cell. Does that imply that tumor cells promote autophagy in neighboring normal (non-tumor) cells to obtain nutrients for their own growth? To address this question, the authors scored tumor growth under a condition in which they removed an amino acid transporter specifically from tumor cells. Indeed, under these conditions, tumor growth is strongly inhibited. ${ }^{7}$ Similar results were recently reported in another paper in Nature in a model for pancreatic ductal adenocarcinoma (PDAC). ${ }^{10}$ Here, NAA was triggered in stromal (non-tumor) pancreatic stellate cells (PSCs) by tumor cells. This results in breakdown of proteins in and release of amino acids by PSCs. ${ }^{10}$ In particular, Alanine obtained from PSCs serves as an alternative carbon source for PDAC. ${ }^{10}$ Although metabolomic profiling was not performed for Ras ${ }^{V 12}$ scrib, these cells may display a similar amino acid requirement (Figure 1). Combined, these two reports provide strong evidence that tumor cells manipulate the microenvironment by triggering NAA to fuel their own growth.

These studies in Nature provide a possible explanation for another puzzling observation. Prompted by the finding that Kras-induced tumors in mouse models are dependent on autophagy for efficient tumor growth, a group at Pfizer/ Novartis tested a large number of Kras-driven cell lines derived from human cancer patients for a similar growth requirement of autophagy. However, very disappointingly, not a single cell line showed dependence on autophagy for growth. ${ }^{11}$ Obviously, these cell lines were cultured in the absence of a tumor-supporting microenvironment and therefore an effect of NAA was not examined. It is therefore possible that NAA plays a much more significant role for tumor development than previously anticipated. It will be important in future studies to further examine the role of NAA in the tumor microenvironment for growth of human tumors. The use of organoids may be beneficial to address this question.

\section{Conflict of Interest}

The authors declare no conflict of interest.

1. Amaravadi R et al. Genes Dev 2016; 30: 1913-1930.

2. Lin L, Baehrecke EH. Mol Cell Oncol 2015; 2: e985913.

3. Anding AL, Baehrecke EH. Curr Topics Dev Biol 2015; 114: 67-91.

4. Ohsumi Y. Cell Res 2014; 24: 9-23.

5. Lebovitz CB et al. Autophagy 2015; 11: 1668-1687.

6. Perez E et al. Oncogene 2015; 34: 3369-3376.

7. Katheder NS et al. Nature 2017; 541: 417-420.

8. Brumby AM, Richardson HE. EMBO J 2003; 22: 5769-5779.

9. Pagliarini RA, Xu T. Science 2003; 302: 1227-1231.

10. Sousa CM et al. Nature 2016; 536: 479-483.

11. Eng $\mathrm{CH}$ et al. Proc Natl Acad Sci USA 2016; 113: 182-187. 